\title{
A novel technique for detailed and time-efficient combustion modeling of fumigated dual-fuel internal combustion engines
}

\author{
Gilles Decan ${ }^{\mathrm{a}, *}$, Tommaso Lucchini ${ }^{\mathrm{b}}$, Gianluca D'Errico ${ }^{\mathrm{b}}$, Sebastian Verhelst ${ }^{\mathrm{a}, \mathrm{c}}$ \\ ${ }^{a}$ Ghent University, Electromechanical, Systems and Metal Engineering, Sint-Pietersnieuwstraat 41, 9000 Ghent, Belgium \\ ${ }^{b}$ Politecnico Di Milano, Department of Energy, Via Lambruschini 4, 20156 Milano, Italy \\ ${ }^{c}$ Lund University, Department of Energy Sciences, Ole Römers väg 1, 22100 Lund, Sweden
}

\begin{abstract}
The present work details a study of the 3D-modeling of dual-fuel engines, with a specific focus on medium speed marine engines. These operate under the fumigated approach, which means that the second fuel, which replaces most of the diesel fuel, is added in the intake duct. This type of engine requires the least amount of modifications to the current medium speed diesel engine and is therefore an ideal retrofit solution to tackle global warming and local air quality issues. In this work, the operation of such an engine has been modeled, with special care for the combustion modeling. Namely, it comprises both auto-ignition of the diesel pilot, and flame propagation in the premixed fuel-air mixture. A tabulated kinetics approach, currently being used to model diesel operation, has been extended to include this flame propagation. Both a Coherent Flame Model (CFM) and a Flame Surface Wrinkling Model (FSWM) have been implemented to handle this premixed combustion mode. Coupling a flame propagation model to the tabulated chemistry should provide a practical way of capturing both the complex dual-fuel combustion process as the chemistry information, while maintaining an acceptable computation time. Additionally, a tabulated laminar flame speed method has also been implemented. Experimental results were obtained on the operation of a medium-speed engine in diesel, natural gas/diesel dual-fuel and methanol/diesel dual-fuel mode, under varying operating loads and speeds. Results have been compared to the ones obtained from the simulations performed in OpenFOAM with the dedicated combustion modeling technique. It was found that while both FSWM and CFM capture some physical trends, they are currently not able of capturing the dual-fuel combustion process in total. Improvements with regards to the ignition and mixing-controlled combustion modeling are necessary, since the current approach is not able of fully capturing the dual-fuel phenomena. This work however provides insight in the complex combustion process and serves as a basis for further developments. It can also be used as an initial engine development tool for fast calculation and optimization of dual-fuel operation.
\end{abstract}

Keywords: Fumigated dual-fuel engine, Engine optimization, Computational Fluid Dynamics, Combustion modeling, Tabulated kinetics

\footnotetext{
${ }^{*}$ Corresponding author

Email address: gilles.decan@ugent . be (Gilles Decan)
} 


\section{Introduction}

Current global warming and local air quality issues, coupled with recently imposed more stringent emission legislation to tackle those issues, provide the drive for engine manufacturers to further develop their propulsion techniques and clean up the emissions from transportation. Especially in the marine industry, where the internal combustion engine remains the number one technology for propulsion, the development and optimization of new engine technologies is high on the agenda. A possible short-term solution is the conversion of the currently used four-stroke medium-speed diesel engine to fumigated dual-fuel operation. This fairly easy and cost-efficient retrofit is made possible by the injection of a second, low-carbon fuel into the intake port. This fuel, such as natural gas or methanol, then constitutes the main part of the necessary energy content and is pre-mixed with air under lean conditions. This premixed charge is ignited around Top Dead Center (TDC) by a small diesel pilot [1].

The fumigated dual-fuel engine has as main advantage the reduction of $\mathrm{NO}_{\mathrm{X}}$ and soot emissions, due to the reduced amount of diesel that combusts in non-premixed mode. Additionally, this approach allows the introduction of renewable fuels, such as renewable methanol [2], as the premixed fuel. In this way, one can shift away from fossil fuels and reduce the carbon footprint of this technology. However, possible downsides come along with the early fuel injection and mixing, like emissions of unburned hydrocarbons (UHC) or CO, especially under non-optimal engine conditions [1, 3,-8].

It is clear that optimization of such an engine is crucial and that the beneficial aspects should be enhanced, while the downsides reduced. An as lean as possible operation with an as high as possible diesel substitution ratio should be aimed for, without endangering stable operation under varying engine conditions. Hereby, all types of emissions should be reduced. It is in this optimization study that Computational Fluid Dynamics (CFD) can play an important role. Various parameters can be more easily investigated numerically than on an experimental test setup, especially for these large marine engines. This work therefore focuses on accurate dual-fuel combustion modeling, which could be implemented in an engine simulation framework for predictive and optimization purposes.

In recent years, engine combustion modeling has mainly been focused on the inclusion of detailed chemistry into the simulations. These allow an accurate prediction of the combustion and species formation, including the formation of harmful emissions like $\mathrm{NO}_{\mathrm{X}}$. However, these detailed kinetics come at a large increase in computation time, since they are described by a large set of stiff differential equations. On-the-fly reduction techniques like TDAC [9] or multizone methods like CCM [10, 11] offer some reduction in the necessary computational resources. The direct solution of these stiff kinetic schemes in the simulation however remains very time-consuming, especially for practical cases like these medium-speed dual-fuel engines.

A means of including these detailed kinetics at an acceptable increase in computation time is offered by tabulated kinetics [12, 13]. In this approach, the reactions are calculated beforehand using a chemistry solver and a dedicated flame structure. The determined reaction rates and species concentrations are then stored in a table, which can be accessed during the CFD simulation. The correct values are retrieved and fed back into the numerical calculation 
depending on the thermo-chemical state of the system [14-16]. The most well-known method of characterizing the thermo-chemical state is the use of a progress variable, which tracks the evolution of the combustion process, combined with other parameters such as the scalar dissipation rate, unburned temperature, mixture fraction, ... Depending on the used flame structure, different tabulation methods are used. Different flame structures have been implemented in the work of Lucchini et al. [15] and a comparison between the combustion modeling with the tabulated approach and direct integration of the kinetics scheme for constant volume combustion has been made. While both methods gave good predictions regarding combustion aspects such as ignition delay, flame lift-off length, ..., the computation time was significantly reduced by using the tabulated approach compared to direct integration.

These tabulated kinetics have also been implemented into diesel engine simulations by Lucchini et al. [14-16] and Maes et al. [17] and their performance has been analyzed. A good prediction of the engine operation was retrieved with an accurate agreement between experimental and numerical pressure and heat release rate traces. Additionally, the performance of different flame structures and therefore different tabulation methods has been analyzed in [15] for the prediction of diesel engine operation. While differences in ignition delay and lift-off length were found between the different methods, more global combustion parameters such as the heat release rate were in general well predicted by all methods.

Fumigated dual-fuel engines however are expected to mainly combust SI-like, so in a turbulence-controlled manner, with a flame propagating through the premixed air-fuel charge. However, a part of the combustion is still in ignition and mixing-controlled combustion mode, for which the modeling is complicated by the presence of the second fuel. Furthermore, with lower levels of the diesel substitution ratio, the share of this complex mixing-controlled combustion increases. Tabulated kinetics only serve to predict the auto-ignition, which ignites the premixed gas, and other combustion models have to be implemented for the flame propagation. In other works therefore, correlations and simplifications have been used. In older works [18--21] combustion submodels like the SHELL model or the Characteristic Time Scale model (CTC) have been used to predict combustion. These are basic models initially developed for diesel combustion, that have been extended to include a secondary fuel. They however do not include any information on chemical kinetics and do not represent the flame propagation. In more recent works [22--25], these and other combustion submodels are still being used to predict the combustion phenomena. While they provide a computationally fast tool for the prediction of combustion in certain setpoints, they lack insight in the physical processes that are going on inside the engine.

A method of including the more physically accurate flame propagation that is going on inside this type of engines, is the use of the 3-Zones Extended Coherent Flame Model (ECFM3Z) as presented by Colin and Benkenida [26]. This method allows to combine the effects of both a diffusion and a premixed flame and can show good results during dual-fuel operation as illustrated by [24, 27]. However, no chemistry information is included in the combustion model and no prediction of species formation is possible. Other works try to couple both a diffusion flame model and a flame propagation model to calculate dual-fuel operation. In the work of Singh et al. [28] a switch is made from the previously used CTC model to the G-Equation model, based on reaching a certain temperature threshold. 
This CTC model is now only used to calculate the ignition, afterwards the G-Equation takes over and models the flame propagation. It more accurately represents the dual-fuel combustion process, but it does not capture chemistry information.

Moon et al. [29] also use a G-Equation to model the flame propagation. In their work it is however coupled with a Representative Interactive Flamelet (RIF) model to predict the diesel pilot auto-ignition and include chemistry information. While it physically represents the dual-fuel combustion process in an accurate way, and chemistry information is obtained during ignition, there is no coupling between the G-Equation and the chemistry information. No combustion progress tracker is present since a level-set approach is implemented that only distinguishes between burned and unburned gas. Accurate results on the combustion progress with regards to pressure and heat release rate are therefore retrieved, but information on species formation is lost when switching to the main combustion mode. Zhou et al. [30] present a similar approach, where direct integration of the chemistry scheme for the prediction of auto-ignition is coupled to a Lagrangian marker flame propagation method. They use this for the simulation of RCCI engines. Similarly, an accurate and physical prediction of the combustion process is obtained. This is however obtained at an increased computational cost, since direct integration of the chemistry scheme has been implemented. Other works [31,34] implement advanced combustion models for the simulation of dual-fuel engines. They however often use commercial software where these models are implemented behind an interface. No details on how the dual-fuel combustion is modeled is given, which limits the understanding of the actual dual-fuel combustion process.

Inclusion of detailed kinetics into the complete dual-fuel simulations can be of great relevance with regards to engine development and optimization. Doing this by means of tabulated kinetics should allow to maintain an acceptable computation time. Being able to continue tracking the species information during flame propagation is a necessary step here, as it will provide detailed information on engine operation and emission formation, such as $\mathrm{NO}_{\mathrm{X}}$ or $\mathrm{UHC}$ levels. In this work therefore, an initial study of a combined method of tabulated kinetics and a flame propagation model is discussed for the simulation of fumigated dual-fuel engines under a large variety of engine settings. It should provide a means to predict the complex combustion phenomena occurring during this dual-fuel mode, while still maintaining an acceptable computation time. Additionally, it should increase the understanding on the dual-fuel combustion process. It is important that this flame propagation model should be described by a combustion tracker, which should be related to the progress variable used in the tabulated kinetics, to maintain the chemistry information from the table. It was chosen to implement both a Coherent Flame Model (CFM) and the Weller Flame Surface Wrinkling Model (FSWM) to represent the flame propagation in the air-fuel mixture. These two models have been applied to a large set of engine conditions for both diesel/natural gas and diesel/methanol dual-fuel operation. Their performance is compared and analyzed.

In the remainder of this paper, the extensive experimental database that was used to compare the numerical results is presented. Thereafter the main part focuses on the combustion modeling, shortly mentioning the tabulated kinetics approach used for diesel combustion. A larger section is dedicated to the extension of this tabulated kinetics approach to dual-fuel combustion modeling, by including a flame propagation model and the necessary calculation of the lam- 
inar flame speed. This innovative approach should allow to capture all physical trends occurring during the complex dual-fuel combustion mode, while still maintaining the detailed chemistry information of the tabulated kinetics at an acceptable numerical cost. This presented methodology is then tested on a variety of dual-fuel natural gas/diesel and methanol/diesel cases and the performance is analyzed.

\section{Nomenclature}

\section{Abbreviations}

ABDC After Bottom Dead Center

AHHR Apparent Heat Release Rate

ATDC After Top Dead Center

BBDC Before Bottom Dead Center

BTDC Before Top Dead Center

CFD Computational Fluid Dynamics

CFM Coherent Flame Model

CTC Characteristic Time Scale

DEF Diesel Energy Fraction

EGR Exhaust Gas Re-circulation

EVO Exhaust Valve Opening

FSWM Flame Surface Wrinkling Model

IMEP Indicated Mean Effective Pressure

IVC Intake Valve Closing

LHV Lower Heating Value

RANS Reynolds Averaged Navier-Stokes

RIF Representative Interactive Flamelet
RMS Root Mean Square

SOI Start Of Injection

SST Shear Stress Transport

TKE Turbulent Kinetic Energy

UHC Unburned Hydrocarbons

Symbols

$I_{0}$ Stretch (-)

$R_{\eta}$ Kolmogorov Reynolds number (-)

$\Sigma$ Flame surface density $\left(\mathrm{m}^{-1}\right)$

$\Xi$ Flame wrinkling (-)

$\alpha$ Thermal diffusivity $\left(m^{2} s^{-1}\right)$

$\mu$ Dynamic viscosity $(\mathrm{Pa} s)$

$\omega$ Specific dissipation rate $\left(s^{-1}\right)$

$\rho$ Density $\left(k g \mathrm{~m}^{-3}\right)$

$\rho_{u}$ Density unburned gas $\left(\mathrm{kg} \mathrm{m}^{-3}\right)$

$\dot{\mathbf{Q}}$ Source term for the rate of heat release $\left(W \mathrm{~m}^{-3}\right)$

m Mass flow rate $\left(k g s^{-1}\right)$

b Regress variable (-)

C Progress variable (-)

$\mathbf{h}_{\mathbf{u}}$ Unburned gas enthalpy $\left(k g \mathrm{~m}^{2} \mathrm{~s}^{-2}\right)$ 
k Turbulent kinetic energy $\left(m^{2} s^{2}\right)$

P Pressure $(\mathrm{Pa})$

$\mathbf{P}_{\mathbf{u}}$ Unburned gas pressure $(\mathrm{Pa})$

Re Reynolds number (-)

$\mathbf{S}_{\mathbf{u}}$ Unstrained Laminar flame speed $\left(m s^{-1}\right)$

Sc Schmidt number (-)
T Temperature $(K)$

$\mathbf{T}_{\mathbf{u}}$ Unburned gas temperature $(K)$

$\mathbf{U}$ Velocity flow field $\left(m s^{-1}\right)$

u' Turbulent velocity fluctuation $\left(\mathrm{m} \mathrm{s}^{-1}\right)$

$\mathbf{Y}_{\mathbf{k}}$ Species mass fraction (-)

$\mathbf{Z}$ Mixture fraction (-)

\section{Experimental and numerical setup}

\subsection{Experimental database}

An extensive database of engine experiments obtained from tests on a marine-type single-cylinder engine was available. This engine was run in diesel only, diesel/natural gas and diesel/methanol dual-fuel operation. Since our main focus was on the dual-fuel combustion, we will elaborate on the experimental data and its parameters for this operation.

The single cylinder test engine is a WTZ Roßlau medium-speed engine representative of a $1000 \mathrm{~kW}$ six-in-line marine engine. It has been converted to fumigated dual-fuel operation by adding natural gas or methanol injectors to the intake duct. In Table 1 the main parameters of this engine can be seen, while in Figure 1 the geometry is visualized, albeit in the form of the computational mesh that was used during the simulations. This mesh was generated by a semiautomatic Python program [35] to allow a spray-oriented structure around the injector and a zone where layers can be added or removed during mesh motion. These features are indicated by the green arrow and orange line respectively in the figure.

A variety of engine operations were studied. For the diesel/natural gas dual-fuel operation, the engine load and speed have been varied and a summary of the studied operations can be seen in Table 2 and Figure 2, where the latter one also graphically represents the genset and propeller points, characterized by operation at nominal or varying speed respectively. The table lists engine load and speed, the $\lambda$-value of methane, which was used as a representative of natural gas for simplicity, and the engine IMEP for each condition. To completely specify the energy content in the engine, the amount of diesel pilot also has to be quantified. This can be done by stating a Diesel Energy Fraction (DEF), which is calculated as follows:

$$
D E F=\frac{\dot{m}_{D} L H V_{D}}{\dot{m}_{D} L H V_{D}+\dot{m}_{F} L H V_{F}} * 100
$$

Here $L H V$ is the Lower Heating Value of both fuels and $\dot{m}$ is their respective mass flow rate. This DEF represents the energy fraction of diesel pilot and gives an indication of the energy substitution by the premixed fuel. In all studied diesel/natural gas dual-fuel cases, its value lay around $10 \%$. 


\begin{tabular}{|l|l|}
\hline Number of cylinders & 1 \\
\hline Bore & $240 \mathrm{~mm}$ \\
\hline Stroke & $290 \mathrm{~mm}$ \\
\hline Compression ratio & 12.1 \\
\hline Engine speed & $630-1000 \mathrm{rpm}$ \\
\hline Intake Valve Closing (IVC) & $68^{\circ}$ ABDC \\
\hline Exhaust Valve Opening (EVO) & $76^{\circ}$ BBDC \\
\hline Start of injection (SOI) & $10^{\circ}$ BTDC \\
\hline Number of injector holes & 9 \\
\hline Injector hole diameter & $0.33 \mathrm{~mm}$ \\
\hline Mesh cell count & 30605 TDC; 110105 IVC \\
\hline
\end{tabular}

Table 1: Parameters of the single-cylinder test engine

\begin{tabular}{|c|c|c|c|c|}
\hline Case & Load & Speed [rpm] & $\lambda_{\mathbf{C H}_{\mathbf{4}}}$ & IMEP [bar] \\
\hline 2200 & $25 \%$ & 630 & 2.0 & 6.2 \\
\hline 2202 & $50 \%$ & 800 & 1.8 & 9.1 \\
\hline 2204 & $75 \%$ & 910 & 1.8 & 12.3 \\
\hline 2206 & $25 \%$ & 1000 & 2.1 & 5.2 \\
\hline 2208 & $50 \%$ & 1000 & 1.9 & 8.3 \\
\hline 2210 & $75 \%$ & 1000 & 1.9 & 11.4 \\
\hline 2212 & $100 \%$ & 1000 & 1.9 & 14.4 \\
\hline
\end{tabular}

Table 2: Summary of the tested diesel/natural gas dual-fuel conditions

For the diesel/methanol dual-fuel cases, the influence of the methanol substitution ratio was investigated. While engine load and speed were kept constant at $75 \%$ and $1000 \mathrm{rpm}$, the $\lambda_{\mathrm{CH}_{3} \mathrm{OH}}$ and the DEF were changed. Three dual-fuel cases, where the substitution ratio was changed between 40 and $60 \%$, and normal diesel operation were studied.

\subsection{Numerical setup}

During this work, RANS simulations of this engine have been carried out and a k- $\omega$ SST turbulence model with standard parameters was implemented. A comparison with a k- $\epsilon$ model was performed, and no difference in results was obtained. A Eulerian-Lagrangian method was chosen to represent the diesel spray. Additionally, Blob injection, KH-RT [36], Ranz-Marshal [37] and Spalding [38] submodels were chosen to model the transition steps of the Lagrangian droplets to the Eulerian gas phase from the injection, through break-up and heat transfer between phases, to 


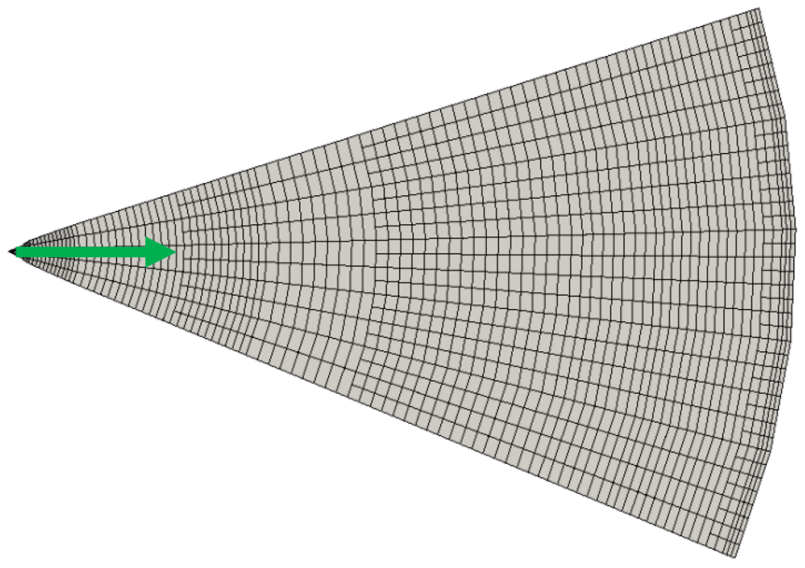

(a) Top view

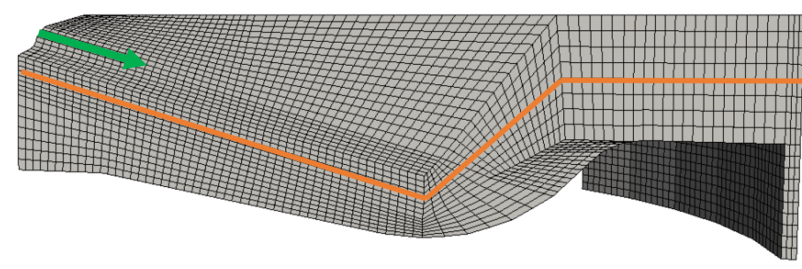

(b) Side view

Figure 1: Computational mesh of the WTZ engine.

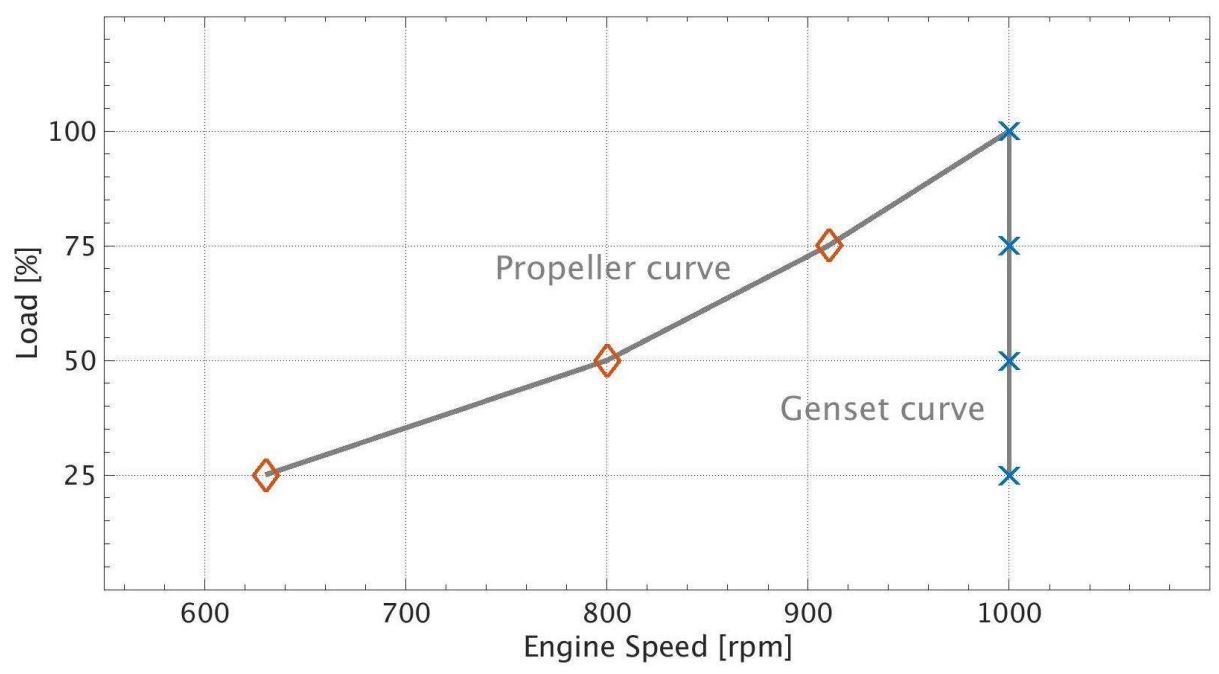

Figure 2: Summary of the the studied dual-fuel operation points

the evaporation of the diesel droplets.

The fields at IVC, the start of the simulation, were initialized based on measurement data. While pressure was initialized directly based on measured values, the trapped mass had to be calculated based on measured mass flow rates. Since there was no valve overlap - to avoid methane slip - the trapped mass of air and natural gas or air and methanol could be calculated and initialized. The flow field at IVC was initialized as a swirling flow, based on an estimated swirl ratio and kinetic energy level, which were later verified by performing gas dynamics simulations of the complete engine. Lastly, diesel fuel was delivered according to the measured mass flow rate profiles. In the next sections, the combustion modeling is elaborated upon, after which results of the performed engine simulations are analyzed and compared. 


\section{Tabulated kinetics for diesel combustion}

The computational approach for the simulation of dual fuel combustion is based on the one presented in [16] for pure diesel engines, where tabulated kinetics was employed to preserve accuracy and computational efficiency at the same time. Only the general steps in this combustion modeling procedure are repeated here, to ensure complete understanding of the methods presented on dual-fuel combustion modeling. For a more detailed description of the tabulated kinetics combustion modeling, one is referred to [16].

\subsection{Table generation}

To include detailed chemistry information, without a large increase in computation time, tabulated kinetics can be used. Auto-ignition and flame propagation are computed in simplified reactors, either in 0-D or 1-D. In these reactor calculations, the chemistry is calculated based on a given chemistry scheme for a range of engine-like conditions. The calculated information, such as the chemistry composition, reaction rate, ignition delay, ... is then stored in a table, which can be accessed by the CFD simulation at runtime.

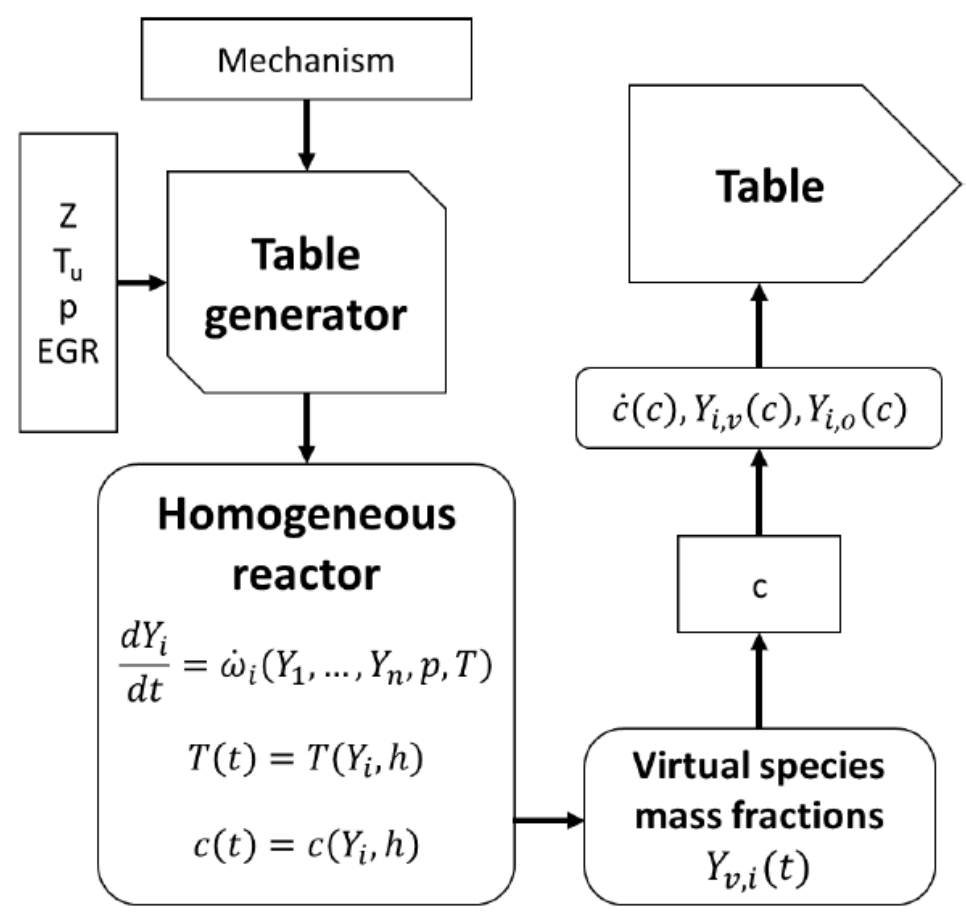

Figure 3: Chemistry calculations and table creation

A graphical representation of this table creation can be seen in Figure 3. Here, the table generator, which was a python script using the Cantera library, is fed with a chemical scheme and a range of possible engine conditions. These are defined by the mixture fraction $Z$ or thus the air-fuel equivalence ratio, the pressure $p$, the unburned gas temperature 
$T_{u}$ and the oxidizer composition or thus the EGR or residuals concentration. Homogeneous reactor calculations are performed and the species concentration, temperature and progress variable are calculated. The definition of the latter one is not straight-forward, and the definition for $C$ being equal to the heat released by combustion as presented by Lehtiniemi et al. [39] was used here. Additionally, the concentration values of all occurring species are also transformed to only the most important ones, such as $\mathrm{O}_{2}, \mathrm{~N}_{2}, \mathrm{CO}, \mathrm{CO}_{2}, f u e l, \mathrm{H}_{2} \mathrm{O}$ and $\mathrm{H}_{2}$ by the virtual species approach as presented in [40]. This reduces the computation time and the table size. The species concentration and progress variable reaction rate $\left(\dot{c}=\frac{c_{i+1}-c_{i}}{t_{i+1}-t_{i}}\right)$ can then be stored as a function of the normalized progress variable

$\left(c=\frac{C-C_{\min }}{C_{\max }-C_{\min }}\right)$. A transport equation for the progress variable, as presented in the next section, can then be solved, to retrieve this chemical information.

\subsection{TWM model}

During the simulations, the Tabulated Well-Mixed (TWM) model was used to model the auto-ignition process. As long as ignition is not reached, the contribution of sub-grid mixing is not considered. Chemical information was directly retrieved from the table, based on the calculated values for the engine conditions, as can be schematically seen in Figure 4 The equations that have to be solved to access the chemistry table are a mixture fraction, a progress variable and an unburned enthalpy equation, seen below in Equations 2. - 4. In these equations, $\rho$ represents the density of the mixture, $\mu$ the laminar or turbulent viscosity, depending on the subscript, $\alpha$ the laminar or turbulent thermal diffusivity and $S c$ the laminar or turbulent Schmidt number. The mixture fraction source term $\dot{S}_{Z}$ in Equation 2 enables the mass transfer through evaporation of the injected liquid diesel spray. Similarly in Equation 4 the source term $\dot{Q}_{s}$ represents the heat transfer due to evaporation. An additional source term due to pressure differences is also present. The progress variable source term $\dot{C}$ controls the combustion rate and can be calculated from the normalized progress variable reaction rate $\dot{c}$, which is retrieved from the table, by multiplying it with the total combustion heat $\left(C_{\max }-C_{\min }\right)$.

$$
\begin{gathered}
\frac{\partial}{\partial t}(\rho Z)+\nabla(\rho \mathbf{U} Z)-\nabla\left(\left(\mu+\mu_{t}\right) \nabla Z\right)=\dot{S}_{Z} \\
\frac{\partial}{\partial t}(\rho C)+\nabla(\rho \mathbf{U} C)-\nabla\left(\left(\frac{\mu}{S c}+\frac{\mu_{t}}{S c_{t}}\right) \nabla C\right)=\rho \dot{C} \\
\frac{\partial}{\partial t}\left(\rho h_{u}\right)+\nabla\left(\rho \mathbf{U} h_{u}\right)-\nabla\left(\left(\alpha+\alpha_{t}\right) \nabla h_{u}\right)=\dot{Q}_{s}+\frac{\rho}{\rho_{u}} \frac{D p}{D t}
\end{gathered}
$$

Solving these equations defines the thermo-chemical state and allows to access the chemistry table. This way, species information and the progress variable reaction rate can be retrieved from the table for every cell in the domain. The diesel engine operation, including detailed chemical information, can therefore be modeled [14-16]. 


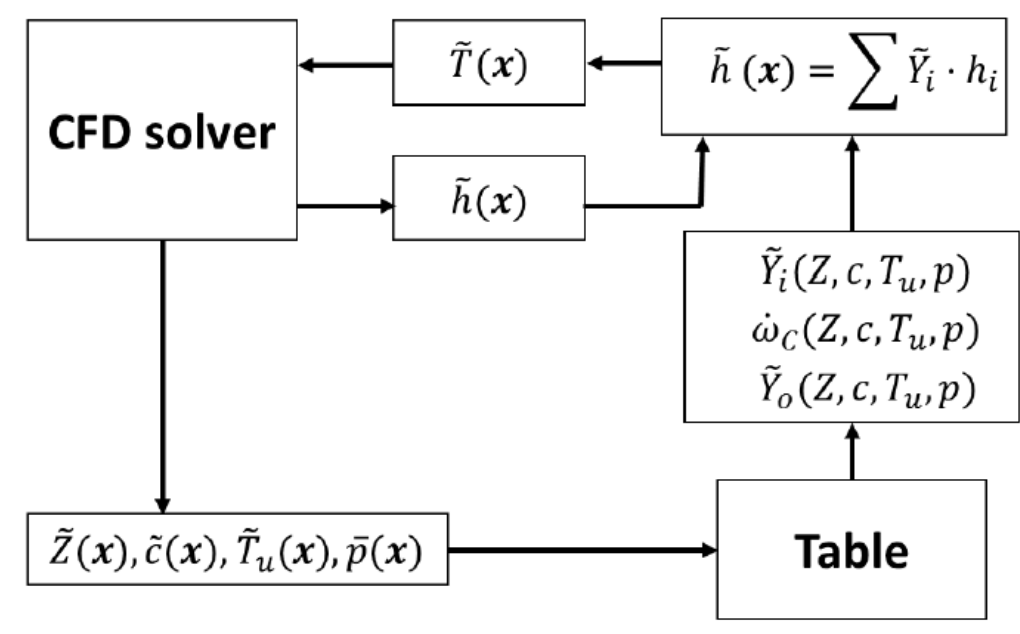

Figure 4: Schematic representation of the TWM approach for diesel combustion modeling

\section{Extension to fumigated dual-fuel operation}

When one wants to model fumigated dual-fuel operation of an engine, tabulated kinetics can still be used, but some adjustments need to be made. To allow proper modeling of the fumigated dual-fuel combustion, the transport equations handling the combustion process need to be altered. In this case, only the progress variable equation undergoes major changes, since terms representing both auto-ignition and flame propagation have to be included. It is important however that this flame propagation model should be described by a combustion tracker, which should be related to the progress variable used in the tabulated kinetics. In this way, chemistry information could still be accessed from the table throughout the complete engine simulation. It was chosen to implement both a Coherent Flame Model (CFM), described by a progress variable, and the Weller Flame Surface Wrinkling Model (FSWM), described by a regress variable, to represent the flame propagation in the air-fuel mixture. Both methods allow access to the pre-calculated chemistry table and are discussed further on.

The mixture fraction Equation 2 remains unchanged but needs to be solved for both fuels. However, since a fumigated dual-fuel approach is being studied, no mixture fraction source term is present for the second fuel. For the same reason - a lack of an evaporation source term from the premixed fuel - Equation 4 can simply be maintained in the mentioned form.

Additionally, since a second fuel is present, changes to the tabulated kinetics are necessary. In the next paragraphs, these changes are mentioned first, followed by the two methods used for the dual-fuel combustion modeling.

\subsection{Modifications to the table generation}

Since for dual-fuel operation, two fuels are present in the engine cylinder, the chemistry table generation should be slightly adapted to include both fuels. An additional input parameter is necessary, namely the mixture fraction of premixed fuel, being $\mathrm{CH}_{4}$ or $\mathrm{CH}_{3} \mathrm{OH}$ in this case. N-heptane was used as the diesel representative. The entire 
discretization of the chemistry table can then be seen in Table 3 For symmetry and uniformity purposes, the premixed fuel has also been considered under rich conditions, even though these never occur during the simulation. Note that due to the addition of premixed fuel, the chemistry table is now around 25 times bigger and its generation now requires more computational effort.

\begin{tabular}{|c|c|}
\hline Pressure [bar] & $20,40,60,90,120,150$ \\
\hline \multirow{4}{*}{ Unburned temperature $[\mathrm{K}]$} & $500-650$ (steps $50 \mathrm{~K}$ ) \\
\hline & 650 - 900 (steps $25 \mathrm{~K}$ ) \\
\hline & $900-1100$ (steps $50 \mathrm{~K}$ ) \\
\hline & $1100-1300$ (steps $100 \mathrm{~K}$ ) \\
\hline \multirow{3}{*}{ Equivalence ratio diesel [-] } & $0.0,0.4-2.0$ (steps 0.1$)$ \\
\hline & $2.0-3.0$ (steps 0.25$)$ \\
\hline & $4.0,5.0$ \\
\hline \multirow{3}{*}{ Equivalence ratio $\mathrm{CH}_{4} / \mathrm{CH}_{3} \mathrm{OH}[-]$} & $0.0,0.4-2.0$ (steps 0.1$)$ \\
\hline & $2.0-3.0$ (steps 0.25 ) \\
\hline & $4.0,5.0$ \\
\hline
\end{tabular}

Table 3: Discretization of the chemistry table

Providing these range of engine conditions, the reactor calculations have been performed. A reduced n-heptane scheme, as described by Ranzi et al. [41], containing 156 species and 3370 reactions was used. This mechanism was found to be best suited for dual-fuel operation. It was detailed enough to also include reactions describing the oxidation of low-carbon fuels such as $\mathrm{CH}_{4}$ and $\mathrm{CH}_{3} \mathrm{OH}$, while still providing a reduction in computation time compared to a complete hydrocarbon scheme [42]. Standard air conditions, consisting of $21 \% \mathrm{O}_{2}$ and $79 \% \mathrm{~N}_{2}$, were applied in the reactor calculations.

Solving the necessary transport equations, as discussed above, allows to access this table again. Only the progress variable equation needs significant changes, to include the effects of the flame propagation. This flame propagation was modeled in two ways, which are discussed in the next paragraphs.

\subsection{Coherent Flame Model}

Since the fumigated dual-fuel combustion mode is a combination of both diffusion controlled combustion of the pilot spray and turbulence controlled combustion of the air-gas mixture, a dedicated flame propagation model has to be implemented. The pilot spray ignition can still be modeled using the tabulated kinetics approach, but a term representing this flame needs to be added to the equations. A first possible solution is the use of the Coherent Flame Model (CFM) as presented in [43]. This CFM approach makes it possible to still control the combustion by a single 
progress variable equation as can be seen in Equation 5 below:

$$
\frac{\partial}{\partial t}(\rho C)+\nabla(\rho \mathbf{U} C)-\nabla\left(\left(\frac{\mu}{S c}+\frac{\mu_{t}}{S c_{t}}\right) \nabla C\right)=\frac{Z_{1}}{Z_{1}+Z_{2}} \rho \dot{C}+\frac{Z_{2}}{Z_{1}+Z_{2}} \rho_{u} S \Sigma\left(C_{\max }-C_{\min }\right)
$$

In this equation, the right hand side now shows two source terms, one for the diesel pilot ignition and one for the flame propagation. They have been scaled according to the mass fractions of the respective diesel or premixed fuel. In this flame propagation source term, $\rho_{u}$ represents the unburned gas density, $S$ the laminar flame speed, which is discussed later on, and $\Sigma$ the flame surface density, which is defined as the ratio between flame surface and cell volume in each cell. It is this term that captures the turbulent effects on the flame and that is modeled in the Coherent Flame Model. Lastly, a multiplication by the $\left(C_{\max }-C_{\min }\right)$ term is necessary since the equation is solved for the non-normalized progress variable. Still controlling the combustion progress by a progress variable equation in this way, lets one continue to use the tabulated kinetics for the detailed chemistry information. After determining the progress variable, species and emission information could still be retrieved.

When modeling the flame surface density, it can on the one hand be done by its own transport equation, a so-called 2-equation model. However, for simplicity, an equilibrium approach was chosen, a so-called 1-equation model, and the flame surface density is calculated from its equilibrium value. Differences with the 2-equation approach were rather limited based on a first comparison, but further investigation of this 2-equation approach will be considered in future work. The equilibrium flame surface density $\Sigma_{\text {equi }}$ was chosen to be calculated by the CFM- 2 method presented by Choi and Huh [44]:

$$
\Sigma_{\text {equi }}=\frac{\alpha u^{\prime} / l_{t c}}{\beta S_{u}}(C(1-C)) \frac{\rho}{\rho_{u}}
$$

where $\alpha$ and $\beta$ are constants chosen at values of 6 and 1 respectively, as those gave the best prediction over a broad range of conditions. $l_{t c}$ was chosen equal to $0.01 \mathrm{~m}$, as a representative of the magnitude of the turbulent length scale of this large marine engine. $u^{\prime}$ is the RMS turbulent velocity fluctuation and $S_{u}$ the unstrained laminar flame speed, which controls the flame propagation. From the inclusion of this $u^{\prime}$, it is directly clear that the turbulent effects on the flame propagation are captured by this $\Sigma$-factor, which is included in the source term in Equation 5 Since RANS simulations were performed, $u^{\prime}$ can be retrieved from the turbulent kinetic energy $k$ by:

$$
u^{\prime}=\sqrt{\frac{2 k}{3}}
$$

\subsection{Weller Flame Surface Wrinkling Model}

The downside of the CFM approach is that the reaction rate $\rho_{u} S \Sigma$ is explicitly solved in the transport equation, which can cause instabilities. To allow a fully implicit solution and improve simulation stability, a different model, proposed by Weller [45], was considered. One then has to switch to a regress variable equation, where the flame propagation is modeled by the Weller Flame Surface Wrinkling Model (FSWM). The benefit of the CFM method is that the combustion progress is controlled by a single equation; this is now no longer possible. With this Weller model two equations are now necessary to model the combustion progress, a progress variable equation to handle the diesel 
pilot ignition and a regress variable equation for the flame propagation. A well-timed switch between the two modes also has to be implemented. These two equations can be seen in Equations 8 and 9 [45-47] below, where Equation 8 is the same as Equation 3 since it only represents the diesel ignition.

$$
\begin{gathered}
\frac{\partial}{\partial t}(\rho C)+\nabla(\rho \mathbf{U} C)-\nabla\left(\left(\frac{\mu}{S c}+\frac{\mu_{t}}{S c_{t}}\right) \nabla C\right)=\rho \dot{C} \\
\frac{\partial}{\partial t}(\rho b)+\nabla(\rho \mathbf{U} b)-\nabla\left(\left(\frac{\mu}{S c}+\frac{\mu_{t}}{S c_{t}}\right) \nabla b\right)=\rho_{u} S \Xi|\nabla b|+\dot{\omega}_{i g n}
\end{gathered}
$$

In these equations, $b$ represents the regress variable, which is equal to 1 in the unburned mixture and 0 in the burned gas. It behaves inversely compared to the normalized progress variable and is therefore equal to $1-c$. The $\Xi$ in the flame propagation source term represents the flame wrinkling and is equal to the ratio of turbulent to laminar flame speed $\frac{S_{t}}{S_{l}}$. The additional source term $\dot{\omega}_{i g n}$ is there to take the ignition effect of the diesel pilot spray into account.

The flame wrinkle factor $\Xi$, which captures the turbulent effects on the flame, can, similarly to the flame surface density $\Sigma$, be modeled by a transport equation or an equilibrium approach. For simplicity reasons, an equilibrium approach was implemented. The flame wrinkling factor $\Xi$ is modeled using Equation 10 [45]. One can see dependencies on both the regress variable $b$ and on $\Xi^{*}$, which in its turn again depends on the equilibrium wrinkling factor $\Xi_{\text {equi }}^{*}$. The dependency on the regress variable is controlled by a tuning constant $S_{\Xi}$. When equal to 0 , the wrinkling is uniform over the flame, while for larger values, it grows over the flame similarly to the turbulence distribution [48]. For simplicity reasons, $S_{\Xi}$ was set to zero in this work and the influence of the regress variable on the flame wrinkling factor was not taken into account.

$$
\Xi=1+\left[1+2 S_{\Xi}(0.5-b)\right]\left(\Xi^{*}-1\right)
$$

The determination of $\Xi^{*}$ follows the same procedure as presented in [48, 49]. It relates $\Xi^{*}$ to the equilibrium value through a stretch factor $I_{0}$ and a laminar to turbulent transition factor $f$. The equilibrium value $\Xi_{\text {equi }}^{*}$ is then given as a function of the Kolmogorov Reynolds number $R_{\eta}$, the RMS turbulence velocity fluctuation $u^{\prime}$ and the unstrained laminar flame speed $S_{u}$. Again, this $u^{\prime}$ directly includes the turbulent effects into the calculation of the $\Xi$-factor, which is in its turn included in the source term of Equation 9 , to capture the complete turbulent flame propagation. A tuning constant $\Xi_{\text {coef }}$ is also present. These relations can be seen in Equations 11 and 12 below.

$$
\begin{aligned}
& \Xi^{*}=I_{0}+I_{0}^{1 / 2} f\left(\Xi_{\text {equi }}^{*}-1\right) \\
& \Xi_{\text {equi }}^{*}=1+\Xi_{\text {coef }} \sqrt{\frac{u^{\prime}}{S_{u}}} R_{\eta}
\end{aligned}
$$

The calculation of the stretch factor and the laminar to turbulent transition factor is the same as in [48, 49] and is not repeated here. The interested reader is referred to their work. The tuning constant $\Xi_{c o e f}$ was found to be an important parameter in the accurate prediction of the flame propagation. It was chosen equal to 0.47 to best match experimental data over a broad range of engine conditions. 
Considering the switch between progress variable and regress variable equation, a transition value for the progress variable $c_{\text {trans }}$ has to be determined. It should allow enough time for the main part of diesel to be ignited and it was therefore chosen equal to 0.9 . When this threshold is reached in a cell, transition to the regress variable equation is made.

During this transition, not only that single cell should be considered to be ignited, but a broader range needs to be determined. All cells in a certain progress variable range are deemed to be igniting. This range is determined by $c_{\text {trans }}$ at its upper limit and another threshold value $c_{\text {threshold }}$ at its lower limit. This latter constant was chosen equal to 0.7 . These igniting cells then contribute to one ignition kernel and make up the ignition source term $\dot{\omega}_{i g n}$ in the regress variable equation 9 For these cells, $\dot{\omega}_{i g n}$ is calculated based on a user-defined ignition strength $C_{s}$ and duration $\Delta t_{i g n}$ given by:

$$
\dot{\omega}_{i g n}=\frac{C_{s} \rho_{u} b}{\Delta t_{i g n}}
$$

Appropriate values for these parameters should be chosen to allow the complete ignition kernel to properly ignite. In this work, they were chosen at values of 0.5 and $5 \cdot 10^{-3} \mathrm{~s}$ respectively.

A downside of this Weller approach, which one can already expect, is that, when the switch to the flame propagation mode is made, no non-premixed combustion is present anymore. A certain zone around the first ignited cell is established to be igniting, and treated as an ignition source for the flame. However, other diesel zones, that were soon to ignite, are now no longer able to. This transition can be delayed by including another switching criterion, besides the $c_{\text {trans }}$-value. A level for the amount of mass that has to be ignited before the switch is made can be included, extending the non-premixed combustion up to a certain amount of mass. The influence of this additional criterion is evaluated further on. The CFM approach on the other hand, due to the inclusion of both correctly scaled source terms into a single equation, should allow ignition and combustion of all diesel, even when flame propagation has already started. When analyzing the results further on, an initial assessment of these trends is given and improvements are proposed.

\subsection{Laminar flame speed modeling}

Both approaches that model the flame propagation in the air-gas mixture require the laminar flame speed to be determined. A submodel that calculates it based on the in-cylinder conditions is therefore necessary. Tabulated values for the laminar flame speed calculated from chemical kinetics, were used. Using the chemical kinetics software Cantera, similar to the table creation described in Section 3.1. and given an appropriate kinetics scheme for the premixed fuel, laminar flame speed values can be pre-calculated under a wide range of operating conditions and stored in a table. The presence and influence of diesel fuel was not included in this calculation, to reduce the computation time, and was deemed acceptable since the main flame propagation occurs in the premixed zone where no diesel is present. The GRI3.0 mechanism [50] was used to calculate the laminar flame speed in air- $\mathrm{CH}_{4}$ mixtures, while a complete C1-C3 mechanism [42] was used for methanol-air mixtures. Compared to other methods for the determination of the laminar flame speed, such as the use of correlations, this should give more realistic results. Especially at elevated 
engine pressures and temperatures, for which these correlations were generally not validated, the laminar flame speed should be more accurately captured by using tabulated values. This is verified by Figure 5 where tabulated values and values obtained with the Gülder correlation [51] are compared under engine conditions. The mentioned results in the next paragraph were then obtained by using tabulated values of this pre-calculated laminar flame speed.
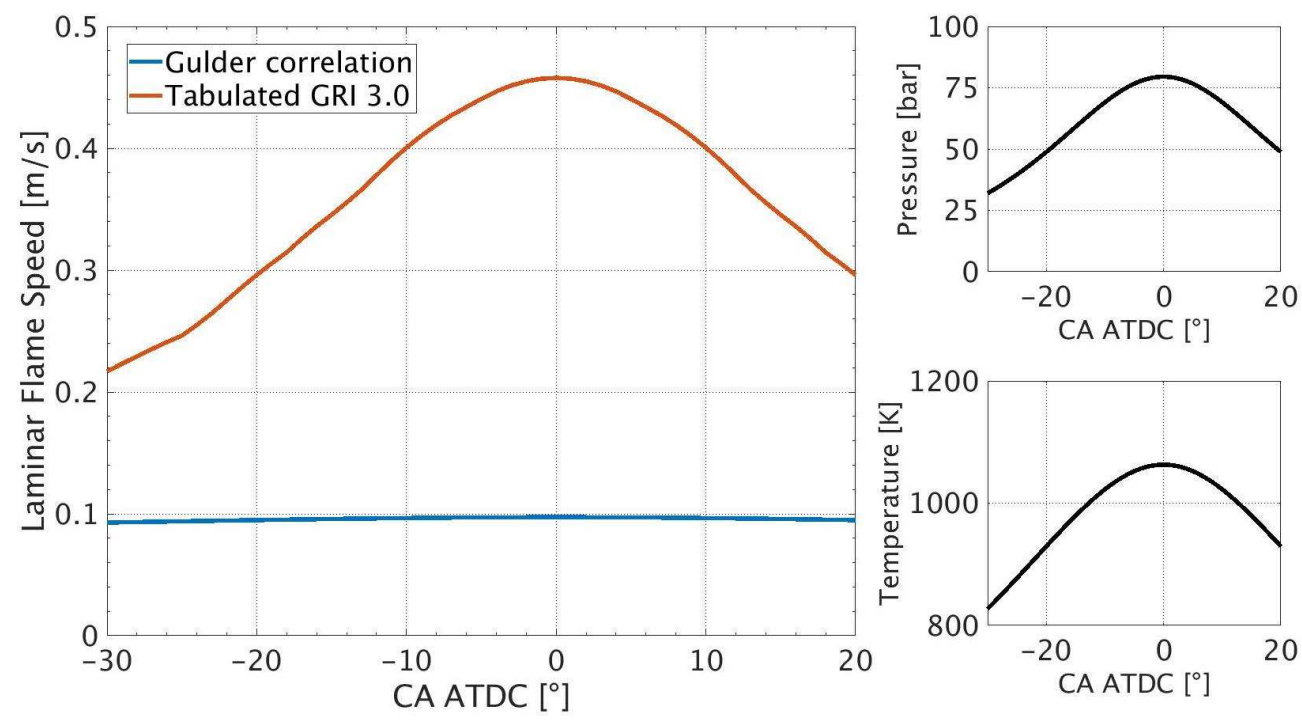

Figure 5: Laminar Flame Speed comparison between the Gülder correlation and tabulated values using the GRI3.0 mechanism; under varying engine pressure and temperature and a constant $\mathrm{CH}_{4}$ equivalence ratio of 0.5

\section{Results and discussion}

The combustion modeling has been implemented in the open-source software OpenFOAM ${ }^{\circledR}$, which has then been used to perform RANS simulations of the closed cycle of the studied engine. Diesel only, diesel/methane dual-fuel and $\mathrm{diesel} / \mathrm{methanol}$ dual-fuel operation of this engine was studied. However, since the main focus of this work was on the dual-fuel combustion modeling, diesel simulations were only quickly performed to validate our approach. Thereafter, the switch to the dual-fuel results was made and results with the presented models were analyzed and compared.

\subsection{Diesel results}

In Figure 6 the pressure and AHHR traces for two diesel test cases, one at full load at $1000 \mathrm{rpm}$ and one at 25\% load at $630 \mathrm{rpm}$, are shown. A good overlap between experimental and numerical trends is observed. It is clear that the tabulated kinetics method is capable of predicting diesel combustion trends, as was also presented in [14[16]. Improvements can however still be obtained, for example in ignition delay prediction, by switching to other flame structures and including sub-grid mixing effects. However, start of injection was not that well-defined for the studied engine, since a pump-line-nozzle system was used. This could therefore already explain some of the observed 
deviations. Especially for this work, where the focus was on dual-fuel combustion, the obtained results were deemed sufficient.

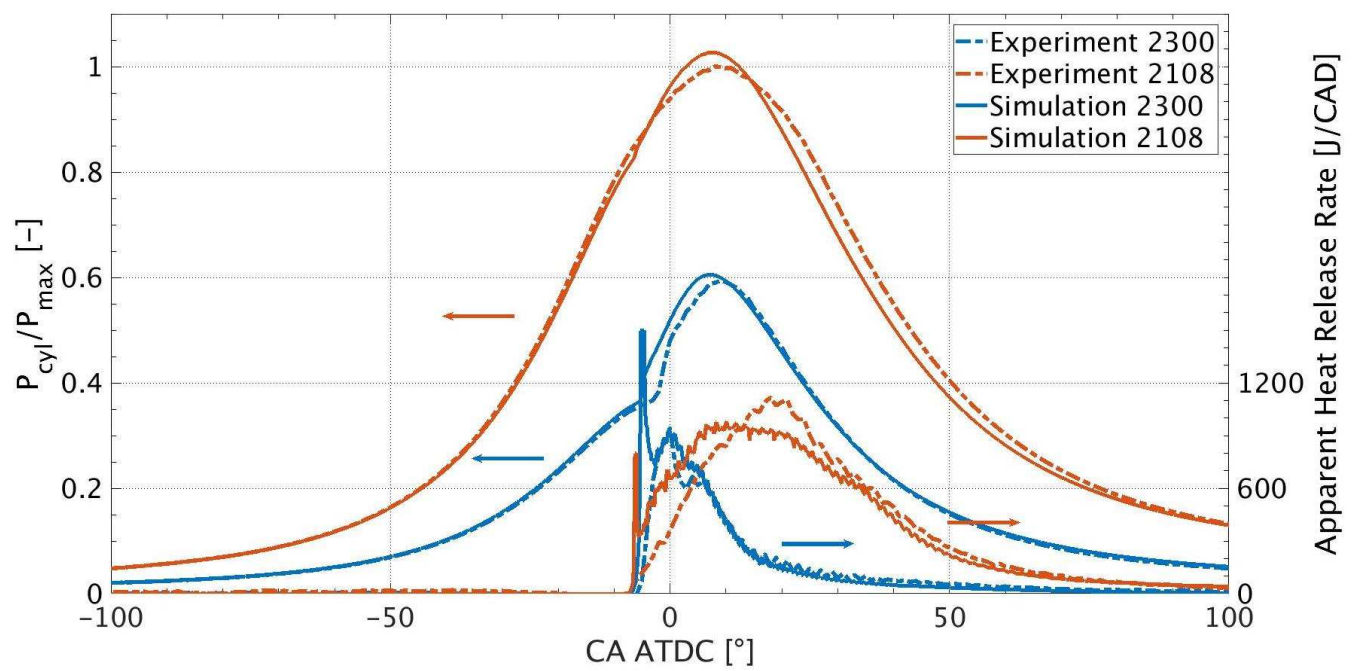

Figure 6: Pressure and AHHR trace for diesel case 2108, 100\% load @ 1000 rpm, in orange and diesel case 2300, 25\% load @ 630 rpm, in blue.

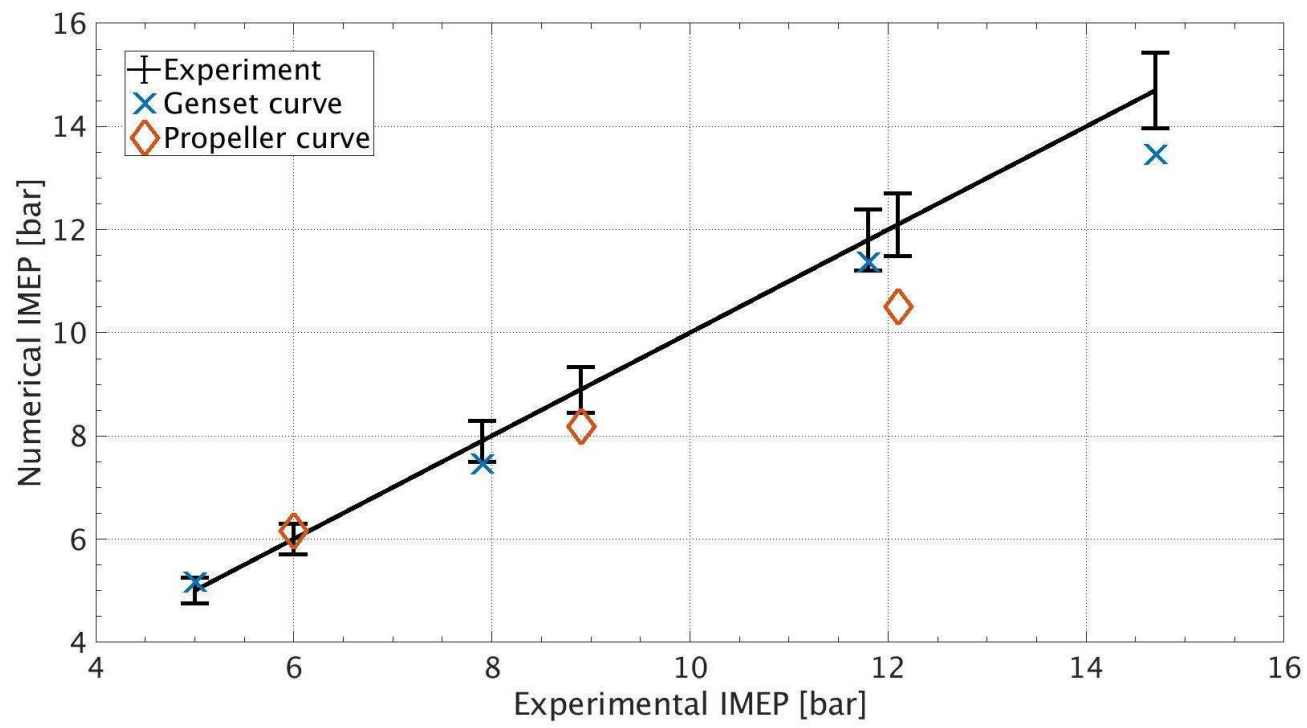

Figure 7: Predicted IMEP values of diesel operation for the studied generator and propeller operating points

The general performance of the diesel simulations for these medium-speed marine engines has been analyzed by the prediction of the IMEP-values for the generator and propeller operating points, which can be seen in Figure 7. A good prediction of the experimentally found IMEP-values is found. Only the two highest loads represent an underprediction of the engine IMEP, which was due to a too short ignition delay and a too early initial pressure rise. However, as was already mentioned, a slight change in start of injection could already improve this. The 
presented results were found satisfactory which led to the conclusion that the current model is capable of predicting diesel operation of these medium-speed marine engines. It can therefore already serve as a development tool for this operation. The extension to the modeling of dual-fuel operation and the possible development and optimization will be discussed in the next sections.

\subsection{Diesel/natural gas dual-fuel results}

The main analysis on the dual-fuel combustion modeling and the evaluation of the performance of the presented modeling techniques will be presented for dual-fuel diesel/ $\mathrm{CH}_{4}$ operation. For this operation mode, measurements with varying load and speed were done, which enabled an analysis of complete engine operation, both in genset and propeller mode. Dual-fuel diesel/methanol simulations have also been performed, but only a single load point was available. Results are therefore only briefly mentioned in the next section, to showcase the applicability of the currently presented method to different premixed fuels.

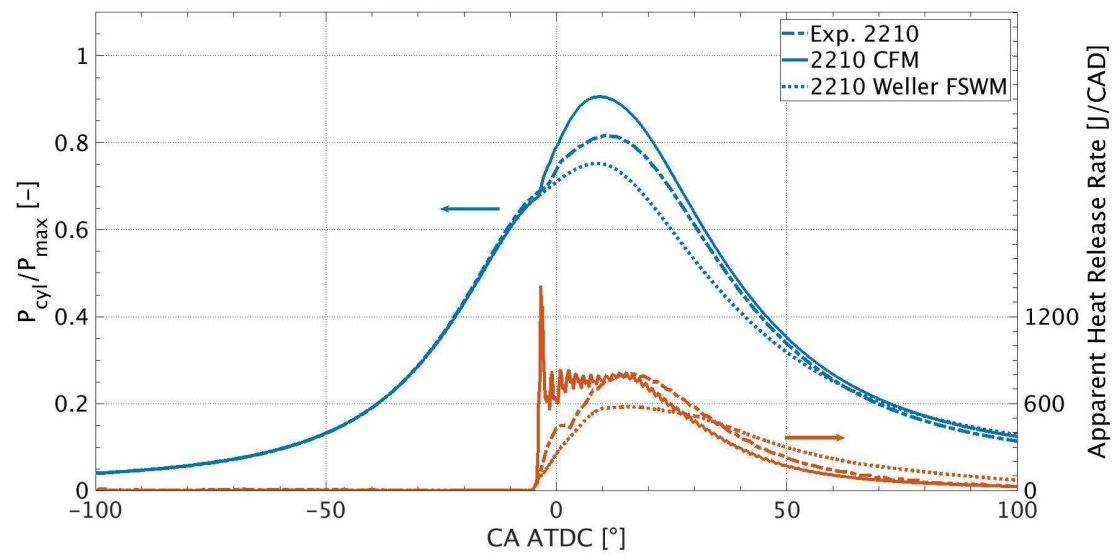

(a) Case 2210: 75\% load @ 1000 rpm

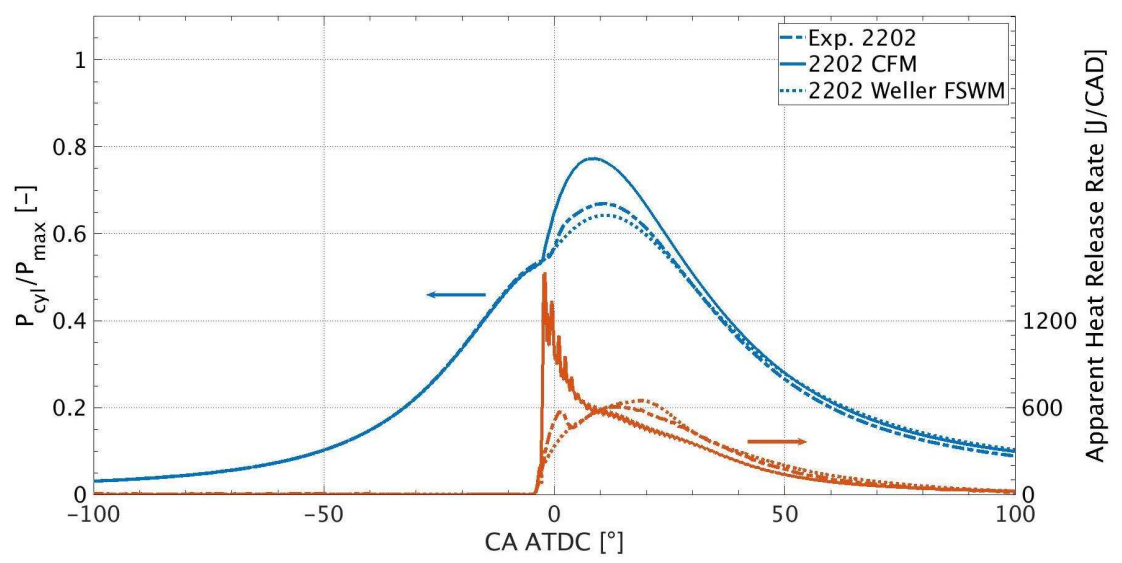

(b) Case 2202: 50\% load @ 800 rpm

Figure 8: Results of the dual-fuel engine for two operational points. The pressure is given in blue, while the AHRR traces are orange. 
In Figure 8, results from two representative dual-fuel cases can be seen, one operating at 50\% load at $800 \mathrm{rpm}$ and one at $75 \%$ load at nominal speed of $1000 \mathrm{rpm}$. For these cases, both pressure and apparent heat release rate as a function of engine crank angle is given, which allows to compare the performance of both the CFM and the Weller model to the experimental results. From these graphs, some general trends are already clear. It is clear that simulations with the CFM model all result in an overprediction of the peak pressure, due to a high pressure rise rate at start of combustion. The results from the Weller FSWM on the other hand behave almost in the opposite manner. All cases represent an SI-like behavior, with smoother pressure and AHRR traces, and an underprediction of the peak pressure. No initial pressure rise at start of combustion is retrieved. To analyze this behavior in more detail, one can have a look at a detailed graph of the combustion for another operating point given in Figure 9 for which the trends were the clearest. This graph is split in three zones, to analyze the complex dual-fuel combustion behavior in these three regions.

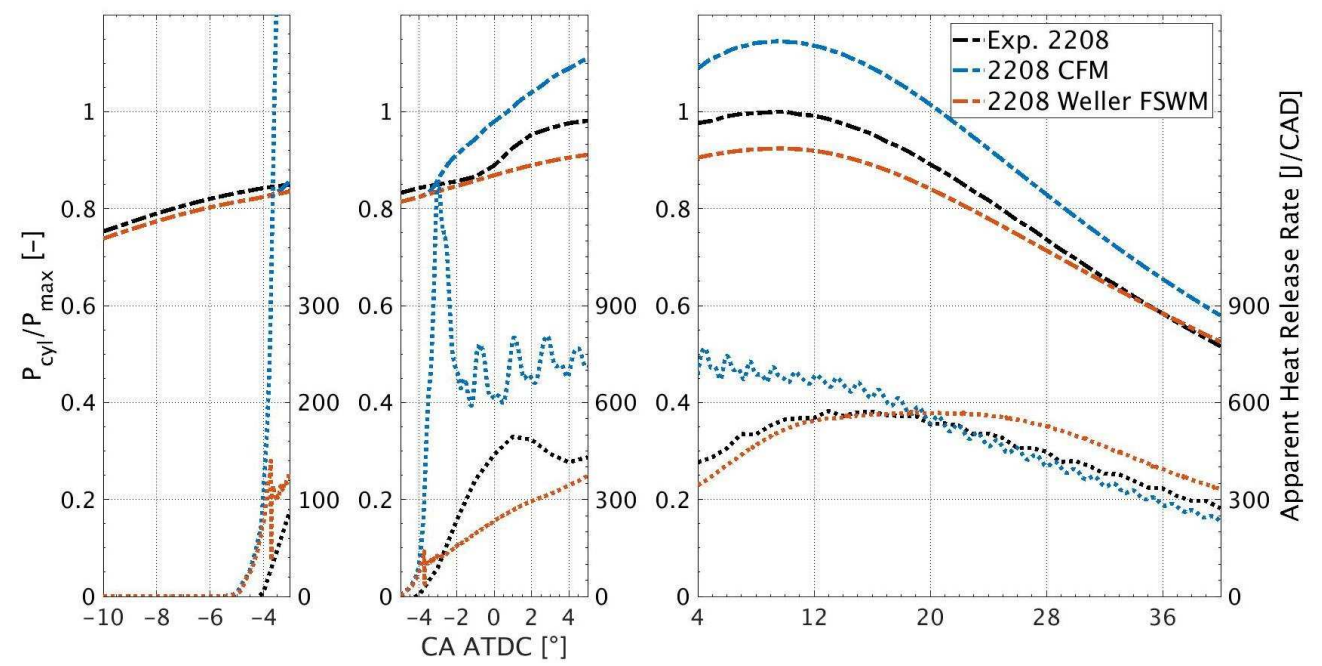

Figure 9: Detailed graph of the pressure and AHHR trace for case 2208, 50\% load @ $1000 \mathrm{rpm}$. The three different combustion phases have been highlighted: ignition, a transition phase and the main flame propagation.

Looking at the first part of the figure, it is clear that for both models, some improvement can be made in the description of the ignition delay and the diesel auto-ignition. A switch to more advanced tabulated kinetics methods as described in [14, 15] or to a RIF method for diesel ignition can hold some improvement. These improved ignition methods will be the focus of future work. Additionally, small deviations in the actual start of injection, which are likely to occur due to the pump-line-nozzle system, could also improve the correspondence. This was verified by delaying the start of injection by $1 \mathrm{CAD}$, which then resulted in some improvements in the ignition delay prediction.

The authors believe that the main improvement can be found in the zone where both diesel is still igniting and the flame starts propagating; a so called transition zone between the two modes which is represented in detail in part two of Figure 9. The limitations of the current Weller model do not allow this combination of combustion modes to be 
accurately predicted, which results in an underprediction of the heat release rate, and therefore also an underestimation of the initial pressure rise. This can however be counteracted by adding the additional ignited mass criterion that has to be met when switching from the progress to the regress variable equation. Instead of switching when the progress variable reaches a threshold value of 0.9 , now also a certain amount of fuel mass, for the FSWM in particular $40 \%$ of the DEF, should be ignited. Additionally, since the diesel ignition will now be active for a longer time, the strength of the ignition term in the regress variable equation is scaled down by reducing $C_{s}$ from 0.5 to 0.2 . As can be seen in Figure 10, it is clear that the magnitude of the ignition and therefore the initial pressure rise rate is now more accurately captured. Even though the combustion phenomena going on after the ignition in this so-called transition zone, are still not captured, good global pressure and heat release rate trends are obtained. These trends were also observed with other operating points, when implementing a switching criterion of $40 \%$ of the DEF. This correction could allow the current numerically fast TWM tabulation method coupled with the FSWM model to be usable for dual-fuel engine development, for example for IMEP and efficiency predictions. The influence on emission formation rates however remains unknown and is the focus of future studies. Further investigation remains necessary and improvements should be analyzed for a variety of engine conditions.

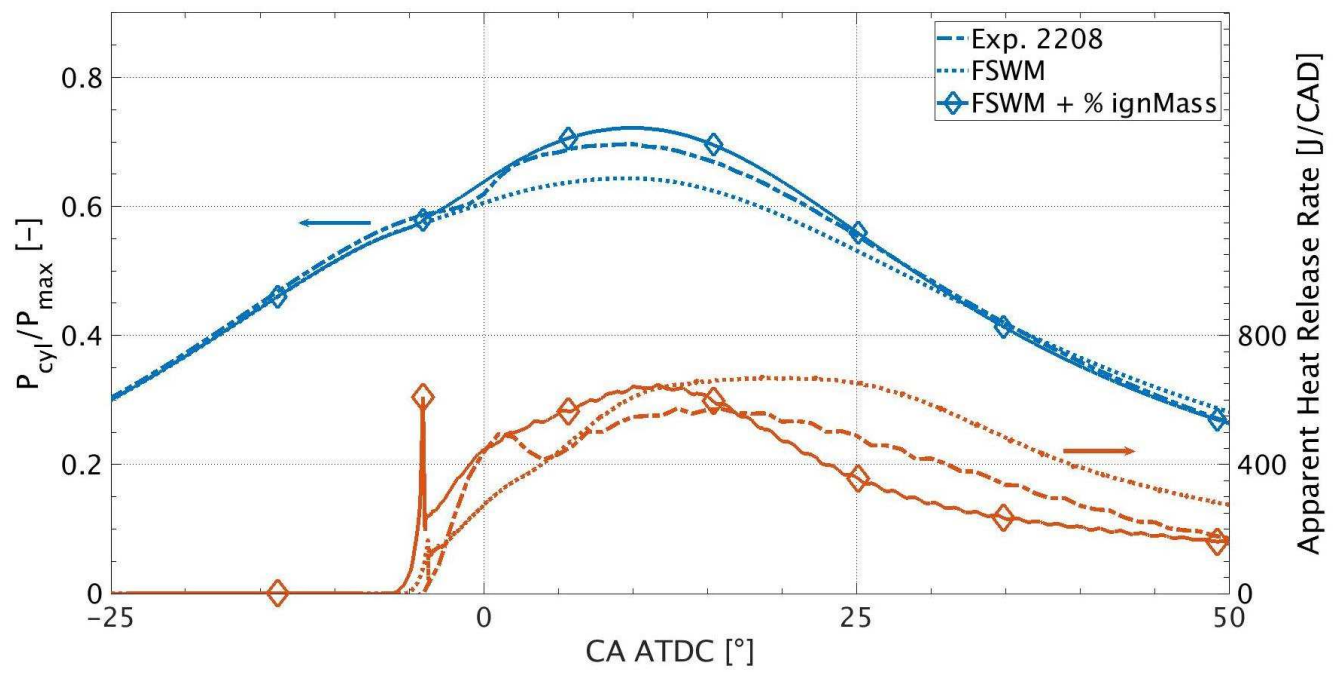

Figure 10: Graph of the pressure and AHHR traces for case 2208, 50\% load @ $1000 \mathrm{rpm}$, obtained with an additional criterion for the transfer from tabulated kinetics to flame propagation.

The CFM model on the other hand does enable this combustion mode partly, as it includes both ignition and flame propagation terms in Equation 5 . The experimentally observed trend of a continuing diesel auto-ignition and combustion, after the ignition delay, which is then followed by the flame propagation, is retrieved. It is however clear that during the transition zone, the actually occurring combustion mode is much more complex than the simple addition of two different modes, since a large overshoot in peak pressure and heat release rate is obtained. The combustion that is occurring at that time does not resemble the predominant features of either a mixing-controlled or 
a turbulence-controlled flame; clearly some complex combustion mode, in between the theoretical limits of mixingand turbulence-controlled combustion, is occurring. A more advanced combustion model to simulate this physical behavior should be investigated. Trying to reduce the large overshoot in heat release rate and pressure, by switching off the diesel ignition source term after a similar threshold of ignited mass is reached, as was introduced for the FSWM simulations, is also analyzed for this CFM model. Improved results are obtained as can be seen in Figure 11 and a clearer flame propagation part is obtained after the switch. Further analysis of this correction however still has to be conducted and its influence under a variety of engine conditions has to be studied.

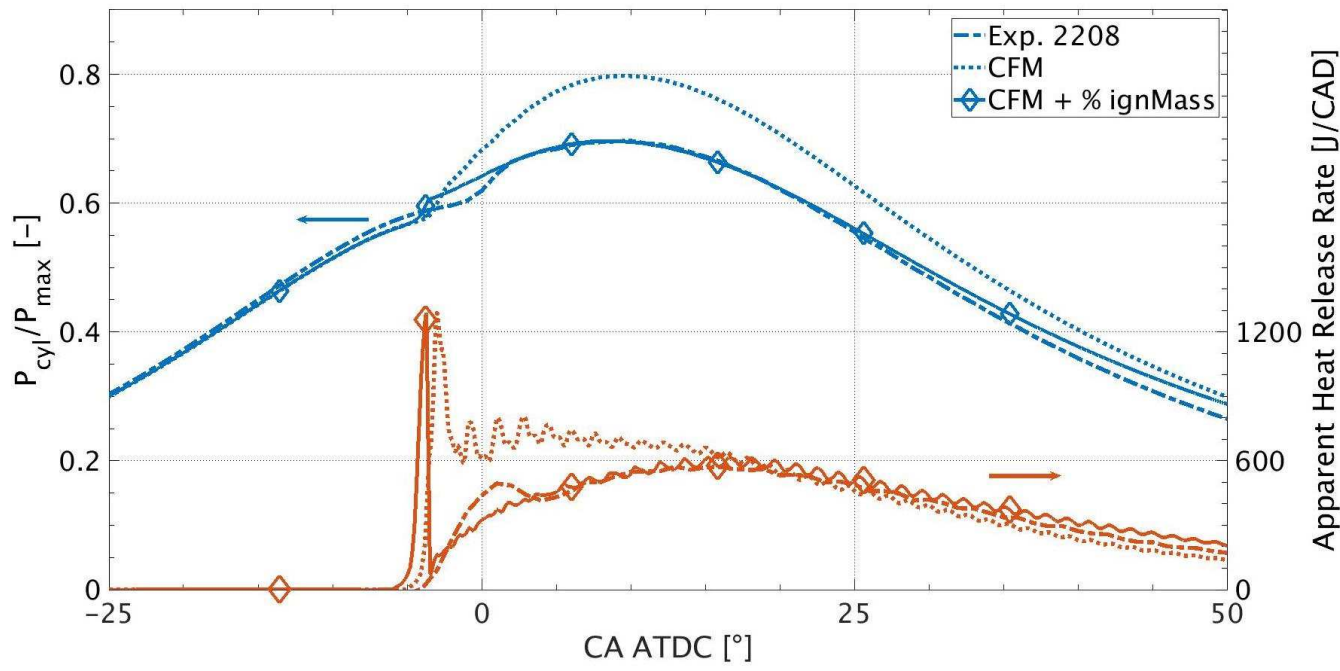

Figure 11: Graph of the pressure and AHHR traces for case 2208, 50\% load @ $1000 \mathrm{rpm}$, obtained with an additional criterion after which the ignition source term, retrieved from the tabulated kinetics, is switched off.

Lastly, if one looks at the main flame propagation part, visualized in the third part of the figure, a good performance is obtained with both methods. The Weller model however performs better compared to the CFM one, especially in the early part where the AHRR retrieved from the CFM model is still dropping due to the previous overshoot during the transition zone. The Weller model already predicts a good rate of combustion there and continues to do so during the remainder of the flame propagation. Additionally, the CFM trace also represents the earlier mentioned instabilities, caused by the explicit implementation of the source term, which is not retrieved with the Weller model.

It is clear that some physics are captured with both models, but that neither succeeds in accurately capturing the complex dual-fuel combustion process, especially with regards to the transition zone between ignition and flame propagation. Advanced methods like for example RIF should be investigated to see if they can hold some improvement. This however does not render the current approach completely useless. First of all, the Weller model accurately predicts the flame propagation but struggles with the combined combustion mode. One can therefore expect much more accurate predictions when an injection strategy is used that ensures a clear separation between the diesel evaporation, the ignition and the flame propagation. For cases where this is not the case, like the ones presented in this 
work, the operation of the Weller FSWM can be extended by including the mentioned criterion of reaching a specified percentage of ignited mass, before switching to the main flame propagation. In this way, acceptable results can be obtained and engine development and optimization, with regards to accurate peak pressure and IMEP predictions, can be conducted. Further investigation in this regard is however necessary, to study the influence on all engine aspects, under varying engine conditions. With regards to the CFM model otherwise, similarly switching of the diesel ignition part can also result in better predictions, as will also be shown for the diesel/methanol simulations presented in the next section. While a simple switch-off of the ignition part does not represent the complexities of the combined combustion mode, it could represent an adequate correction that still enables the use of these fast engine simulations without drastically increasing the required numerical resources. Its influence however needs to be further studied and this investigation, combined with other more advanced tabulation methods to improve the dual-fuel combustion model, will be the focus of future work.

\subsection{Diesel/methanol dual-fuel results}

Diesel/methanol simulations have been conducted as well, to check the applicability of the current model to different premixed fuels, without the need to change the simulation setup or the model constants and parameters. In Figure 12 pressure and AHRR results are presented for a single diesel/methanol case and a similar performance as with diesel/ $/ \mathrm{CH}_{4}$ can be seen.

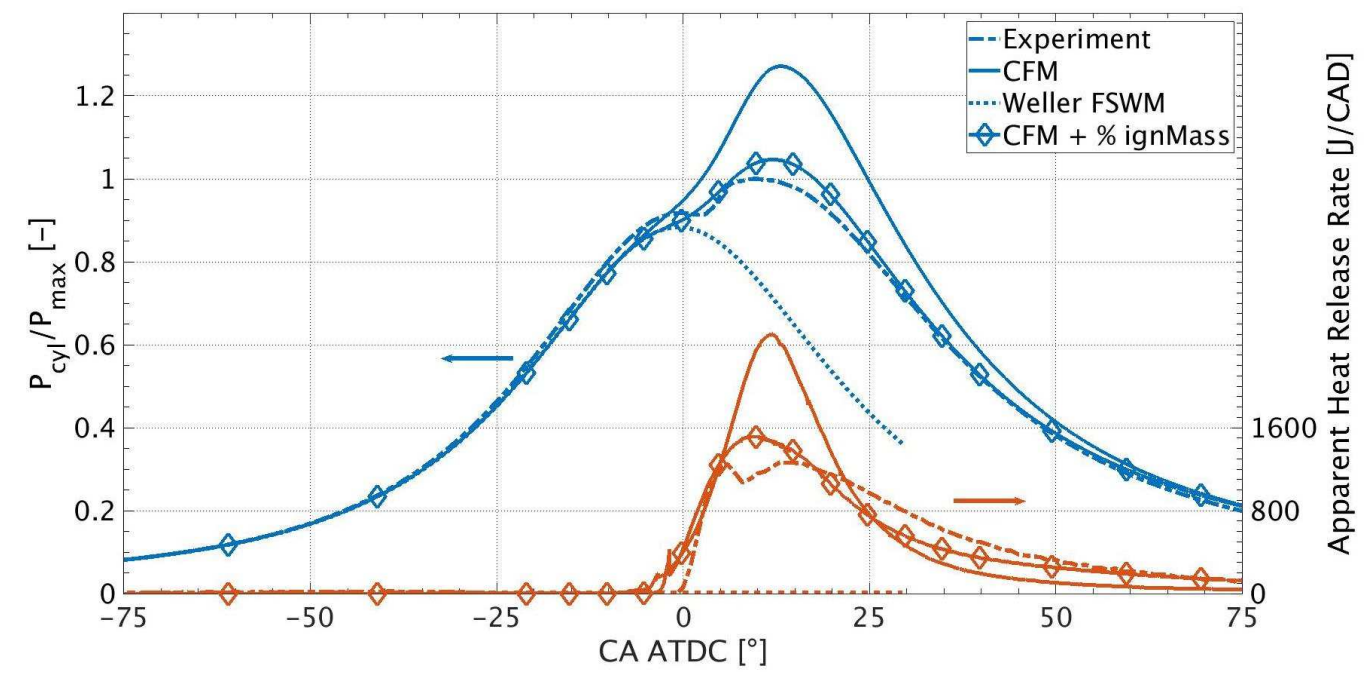

Figure 12: Results of the diesel/methanol dual-fuel engine operating at 75\% load @ $1000 \mathrm{rpm}$. The pressure is given in blue, while the AHRR traces are orange.

Results obtained with the CFM model again show an overshoot in peak pressure, due to an excessively fast heat release during ignition and during the transition zone. However, the overshoot is now even larger when comparing it to the previous results. The authors believe that this is due to the limited amount of premixed fuel, which is only around 50\% in this case, causing the combustion mode to be more of a mix between non-premixed and premixed 
combustion. A large part of combustion now consists of this combination mode between diesel ignition and premixed fuel combustion, making the accurate modeling even more complex and requiring an advanced model. However, to still allow some prediction capabilities for this operating point, using the current approach, switching off the diesel ignition as presented previously, seems necessary for the CFM simulations. Improved in-cylinder pressure predictions are then obtained, which can also be seen in Figure 12

For the Weller FSWM, where the ignition is already switched off after reaching the threshold, results now indicate an additional problem. For this operating point, no combustion is obtained after the initial small diesel ignition. This further indicates that the combustion that is occurring can no longer be described by a succession of ignition and flame propagation and that the current model, which focuses on the flame propagation, fails to capture the combustion. The occurring combustion mode is now clearly a complex combination of mixing-controlled and premixed combustion and an advanced combustion model is necessary to capture this. The Weller model therefore does not seem suited for these simulations, where the diesel energy fraction remains quite high.

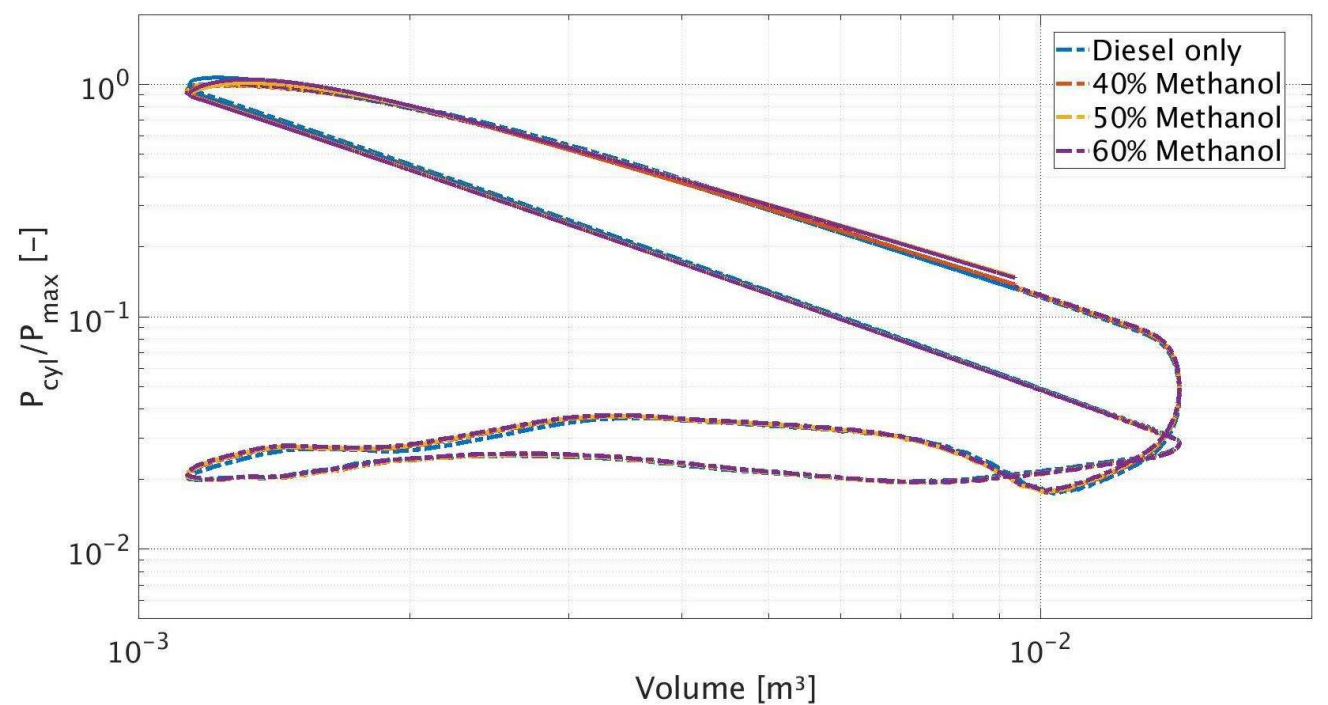

Figure 13: PV-diagrams for the diesel and diesel/methanol operation, investigating the change in the amount of premixed methanol while maintaining the $75 \%$ load operation @ 1000 rpm.

Since the same load point was simulated for these diesel/methanol cases, but with a varying DEF, it is interesting to have a look at the predicted PV-diagrams, obtained from the tuned CFM simulations. These can be seen in Figure 13 Even though not all physical trends are captured, initial engine development can perhaps still be conducted using this method. Therefore, independently from the fuel content, an overlap of the PV-diagrams should be obtained, since the operating points deliver the same amount of work. This is also retrieved in the figure, indicating that the presented method can serve as an initial tool with regards to IMEP or efficiency predictions, and can be the basis for further optimization of this numerically fast coupled tabulated kinetics and flame propagation approach. 


\section{Summary and conclusions}

When dealing with the current global warming and local air quality challenges, engine manufacturers face the need of improving their propulsion technologies. Especially for marine applications, dual-fuel engines seem an interesting and cost-efficient retrofit solution to tackle these issues, without impairing the ship's autonomy. By replacing a large part of diesel by a premixed fuel, they reduce soot and $\mathrm{NO}_{\mathrm{X}}$ emissions while allowing the introduction of renewable fuels into the vessel fleet. When optimizing the operation of these engines, Computational Fluid Dynamics can serve as an important tool.

In this work an improved dual-fuel combustion model, that includes tabulated chemistry, has been presented. A coupled approach between two flame propagation models and a progress variable approach for the simulation of the combustion process has been presented. Both a Coherent Flame Model and a Weller Flame Surface Wrinkling Model have been presented and their performance has been compared. Since they have been described as a function of the progress variable, chemistry information could be accessed from the table during the complete dual-fuel operation. One should therefore be able to track emissions, such as $\mathrm{N}_{\mathrm{X}}$, but information on the unburned methane concentrations, which should be reduced as much as possible, could also be retrieved. This will be investigated in future studies.

Results on both flame propagation methods have been presented. While both of them are able to capture some trends during dual-fuel combustion, neither is able to capture the entire process accurately. While the Weller model presents accurate results on flame propagation, it is limited with respect to the diesel ignition and the combined combustion of non-premixed diesel and premixed main fuel. This is due to the fact that the ignition part can no longer be included when changing to the description of the Weller flame propagation. However, the presented approach could result in good dual-fuel predictions, when the ignition and flame propagation part are more clearly separated. Additionally, including an extra criterion before switching to the flame propagation mode, allows a longer non-premixed combustion phase and results in more accurate global pressure and AHHR trends. The CFM model on the other hand can include this ignition, together with the flame propagation. However, an overshoot of the heat release rate and therefore the pressure rise was obtained for all cases. Further development and improvement of the presented methodology is therefore necessary. These improvements, by investigating possible advanced combustion modes to tackle the complex mixing-controlled combustion found for these engines, as well as the simpler currently presented ad-hoc corrections, will be the focus of future work. The current work as presented in this paper can serve as an initial step in the study of the applicability of these combined tabulated kinetics and flame propagation methods for the simulation of dual-fuel engines, and should be investigated for future improvements.

\section{Acknowledgements}

This research has been funded by Ghent University (Belgium) through GOA project [BOF16/GOA/004]. The authors gratefully acknowledge the financial support. The authors also want to express their sincere appreciation to 
Anglo Belgian Corporation, manufacturer of medium speed engines, and WTZ Roßlau, for the experimental data they provided.

\section{References}

[1] A. Wagemakers, C. Leermakers, Review on the effects of dual-fuel operation, using diesel and gaseous fuels, on emissions and performance, SAE Technical Paper 2012-01-0869 (2012) 1-24doi:10.4271/2012-01-0869

[2] S. Verhelst, J. Turner, L. Sileghem, J. Vancoillie, Methanol as a fuel for internal combustion engines, Prog Energy Combust Sci 70 (2019) 43-88. doi:10.1016/j.pecs.2018.10.001

[3] R. Papagiannakis, D. Hountalas, Combustion and exhaust emission characteristics of a dual fuel compression ignition engine operated with pilot diesel fuel and natural gas, Energy Convers Manag (2004) 1-1\% doi:10.1016/j.enocnman.2004.01.013

[4] J. Egúsquiza, S. Braga, C. Braga, Performance and gaseous emissions characteristics of a natural gas/diesel dual fuel turbocharged and aftercooled engine, J Braz Soc Mech Sci Eng 31 (2009) 142 - 150. doi:10.1590/S1678-58782009000200007

[5] R. Papagiannakis, C. Rakopoulos, D. Hountalas, D. Rakopoulos, Emission characteristics of high speed, dual fuel, compression ignition engine operating in a wide range of natural gas/diesel fuel proportions, Fuel 89 (2010) 1397-1406. doi:10.1016/j.fuel.2009.11.001

[6] V. Macin, V. Bermdez, D. Villalta, L. Soto, Effects of low-pressure egr on gaseous emissions and particle size distribution from a dual-mode dual-fuel (dmdf) concept in a medium-duty engine, Appl Therm Eng 163. doi:10.1016/j.applthermaleng.2019.114245

[7] J. Jung, S. Song, K. Hur, Numerical study on the effects of intake valve timing on performance of a natural gas-diesel dual-fuel engine and multi-objective pareto optimization, Appl Therm Eng 121 (2017) 604-616. doi:10.1016/j . applthermaleng.2017.03.036

[8] L. Wei, P. Geng, A review on natural gas/diesel dual fuel combustion, emissions and performance, Fuel Processing Tech 142 (2016) $264-278$. doi:10.1016/j.fuproc.2015.09.018

[9] F. Contino, H. Jeanmart, T. Lucchini, G. D’Errico, Coupling of in situ adaptive tabulation and dynamic adaptive chemistry: An effective method for solving combustion in engine simulations, Proc Combust Ins doi:10.1016/j.proci.2010.08.002

[10] A. Babajimopoulos, D. Assanis, D. Flowers, S. Aceves, R. Hessel, A fully coupled computational fluid dynamics and multi-zone model with detailed chemical kinetics for the simulation of premixed charge compression ignition engines, Int J Engine Res 6 (5) (2005) $497-512$. doi:10.1243/146808705X30503

[11] M. Raju, M. Wang, M. Dai, W. Piggott, D. Flowers, Acceleration of detailed chemical kinetics using multi-zone modeling for cfd in internal combustion engine simulations, SAE Technical Paper, 2012-01-0135 doi:10.4271/2012-01-0135

[12] T. Poinsot, D. Veynante, Theoretical and Numerical Combustion, 3rd Edition, T. Poinsot, 2012, ISBN: 9782746639904.

[13] N. Peters, Turbulent combustion, 4th Edition, Cambridge University Press, 2006, ISBN: 9780521660822.

[14] T. Lucchini, G. D’Errico, T. Cerri, A. Onorati, G. Hardy, Experimental validation of combustion models for diesel engines based on tabulated kinetics in a wide range of operating conditions, SAE Technical Paper 2017-24-0029 (2017) 1-14doi:10.4271/2017-24-0029

[15] T. Lucchini, G. D’Errico, A. O. A. Frassoldati, A. Stagni, G. Hardy, Modeling non-premixed combustion using tabulated kinetics and different flame structure assumptions, SAE Technical Paper 2017-01-0556 (2017) 1-16 doi : 10.4271/2017-01-0556

[16] T. Lucchini, A. Della Torre, G. D'Errico, A. Onorati, Modeling advanced combustion modes in compression ignition engines with tabulated kinetics, Appl Energy 247 (2019) 537-548. doi:10.1016/j.apenergy.2019.04.062

[17] N. Maes, N. Dam, B. Somers, T. Lucchini, G. D’Errico, G. Hardy, Heavy-duty diesel engine spray combustion processes: Experiments and numerical simulations, SAE Technical Paper 2018-01-1689 (2018) 1-22doi:10.4271/2018-01-1689

[18] Y. Zhang, S. Kong, R. Reitz, Modeling and simulation of a dual fuel (diesel/natural gas) engine with multidimensional cfd, SAE Technical Paper 2003-01-0755 (2003) 1-14doi:10.4271/2003-01-0755

[19] S. Cordiner, V.Rocco, R. Scarcelli, M. Gambino, S. Iannaccone, Experiments and multi-dimensional simulation of dual-fuel diesel/natural gas engines, SAE Technical Paper 2007-24-0124 (2007) 1-18doi :10.4271/2007-24-0124 
[20] S. Cordiner, M. Gambino, S. Iannaccone, V. Rocco, R. Scarcelli, Numerical and experimental analysis of combusiton and exhaust emissions in a dual-fuel diesel/natural gas engine, Energy \& Fuels 22 (2008) 1418-1424. doi:10.1016/ef7004755

[21] S. Singh, S. Kong, R. Reitz, S. Krishnan, K. Midkiff, Modeling and experiments of dual-fuel engine combustion and emissions, SAE Technical Paper 2004-01-0092 (2004) 1-12 doi:10.4271/2004-01-0092

[22] R. Maurya, P. Mishra, Parametric investigation on combustion and emissions characteristics of a dual fuel (natural gas port injection and diesel pilot injection engine using 0-d srm and 3d cfd approach, Fuel 210 (2017) 900-913. doi:10.1016/j.fuel.2017.09.021

[23] M. Cameretti, R. Tuccillo, L. De Simio, S. Iannaccone, U. Ciaravola, A numerical and experimental study of dual fuel diesel engine for different injection timings, Appl Therm Eng 101 (2016) 630-638. doi:10.1016/j.applthermaleng.2015.12.071

[24] J. Shu, J. Fu, J. Liu, L. Zhang, Z. Zhao, Experimental and computational study on the effects of injection timing on thermodynamics, combustion and emission characteristics of a natural gas (ng)-diesel dual fuel engine at low speed and low load, Energy Convers Manag 160 (2018) 426-438. doi:10.1016/j.enconman.2018.01.047

[25] M. Costa, M. La Villetta, N. Massarotti, D. Piazzullo, V. Rocco, Numerical analysis of a compression ignition engine powered in the dual-fuel mode with syngas and biodiesel, Energy 137 (2017) 969-979. doi:10.1016/j.energy .2017.02.160

[26] O. Colin, A. Benkenida, The 3-zones extended coherent flame model (ecfm3z) for computing premixed/diffusion combustion, Oil \& Gas Sci and Tech - Rev IFP 59 (2004) 593-609. doi:10.2516/ogst : 2004043

[27] M. Schiffner, M. Grochowina, T. Sattelmayer, Development of a numerical model for ignition phenomena in a micro pilot ignited dual fuel engine with external mixture formation, Proc ASME 2017 Intern Combust Eng Div Fall Techical Conference (2017) 1-11doi:10.1115/ ICEF2017-3548

[28] S. Singh, L. Liang, S.-C. Kong, R. Reitz, Development of a flame propagation model for dual-fuel partially premixed compression ignition engines, Int J Engine Res 7 (2006) 65-75. doi:10.1243/146808705X7464

[29] S. Moon, S. Chu, T. Nam, K. Min, A study of flow characteristics on the diesel-gasoline dual-fuel combustion by 3-d cfd, SAE Technical Paper 2019-24-0117 (2019) 1-10 doi:10.4271/2019-24-0117

[30] D. Zhou, W. Yang, F. Zhao, J. Li, Dual-fuel rcci engine combustion modeling with detailed chemistry considering flame propagation in partially premixed combustion, Appl Energy 203 (2017) 164-176. doi:10.1016/j .apenergy .2017.06.021

[31] M. C. Cameretti, R. De Robbio, R. Tuccillo, V. Pedrozo, H. Zhao, Integrated cfd-experimental methodology for the study of a dual fuel heavy duty diesel engine, SAE Technical Paper 2019-24-0093 (2019) 1-15 doi:10.4271/2019-24-0093

[32] H. Khatamnejad, B. Jafari, D. Ganji, Investigating the effect of piston bowl geometry on the partially premixed dual fuel combustion engine at low load condition, Eur Phys J Plus 134 (2019) 587-601. doi:10.1140/epjp/i2019-12935-0

[33] Y. Li, H. Li, H. Guo, Y. Li, M. Yao, A numerical investigation on methane combustion and emissions from a natural gas-diesel dual fuel engine using cfd model, Appl Energy 205 (2017) 153-162. doi:10.1016/j .apenergy.2017.07.071

[34] A. Yousefi, M. Birouk, H. Guo, An experimental and numerical study of the effect of diesel injection timing on natural gas/diesel dual-fuel combustion at low load, Fuel 203 (2017) 642-657. doi:10.1016/j.fuel.2017.05.009

[35] T. Lucchini, A. Della Torre, G. D’Errico, G. Montenegro, M. Fiocco, A. Maghbouli, Automatic mesh generation for cfd simulations of direct-injection engines, SAE Technical Paper 2015-01-0376 (2015) 1-16 doi:10.4271/2015-01-0376

[36] M. Patterson, R. Reitz, Modeling the effects of fuel spray characteristics on diesel engine combustion and emission, SAE Technical Paper 980131 (1998) 1-19doi:10.4271/980131

[37] W. Ranz, W. Marschal, Evaporation from drops, Che Eng Prog 48 (1952) 141-146.

[38] D. Spalding, The combustion of liquid fuels, Fourth Symp Combust 4 (1953) 847-864. doi:10.1016/S0082-0784(53)80110-4

[39] H. Lehtiniemi, Y. Zhang, R. Rawat, F. Mauss, Efficient 3-d cfd combustion modeling with transient flamelet models, SAE Technical Paper 2008-01-0957 (2008) 1-23 doi:10.4271/2008-01-0957

[40] G. D’Errico, T. Lucchini, A. Onorati, G. Hardy, Computational fluid dynamics modeling of combustion in heavy-duty diesel engines, Int J Engine Res 16 (1) (2015) 112-124. doi:10.1177/1468087414561276

[41] E. Ranzi, A. Frassoldati, A. Stagni, M. Pelucchi, A. Cuoci, T. Faravelli, Reduced kinetic schemes of complex reaction systems: Fossil and 
biomass-derived transportation fuels, Int J Chem Kin 46 (2014) 512-542. doi : 10.1002/kin.20867

[42] E. Ranzi, A. Frassoldati, R. Grana, A. Cuoci, T. Faravelli, A. Kelly, C. Law, Hierarchical and comparative kinetic modeling of laminar flame speeds of hydrocarbon and oxygenated fuels, Prog Energy Combust Sci 38 (2012) 468-501. doi:10.1016/j.pecs.2012.03.004

[43] J. Duclos, D. Veynante, T. Poinsot, A comparison of flamelet models for premixed turbulent combustion, Combust Flame 95 (1993) $101-117$. doi:10.1016/0010-2180(93)90055-8

[44] C. Choi, K. Huh, Development of a coherent flamelet model for a spark-ignited turbulent premixed flame in a closed vessel, Combust Flame 114 (1998) 336-348. doi:10.1016/S0010-2180(97)00194-6

[45] H. Weller, The development of a new flame area combustion model using conditional averaging, Thermo-Fluids Section Report TF /9307 (1993) 1-60.

[46] G. Tabor, H. Weller, Large eddy simulation of premixed turbulent combustion using $\Xi$ flame surface wrinkling model, Flow Turbulence Combust 72 (2004) 1-27. doi:10.1023/B:APPL.0000014910.06345.fb

[47] H. Weller, G. Tabor, A. Gosman, C. Fureby, Application of a flame-wrinkling les combustion model to a turbulent mixing layer, Symp (Int) Combust 27 (1998) 899-907. doi:10.1016/S0082-0784(98)80487-6

[48] L. Sforza, T. Lucchini, G. Gianetti, G. D’Errico, Development and validation of si combustion models for natural-gas heavy-duty engines, SAE Technical Paper 2019-24-0096 (2019) 1-13 doi:10.4271/2019-24-0096

[49] T. Lucchini, G. D'Errico, D. Paredi, L. Sforza, A. Onorati, Cfd modeling of gas exchange, fuel-air mixing and combustion in gasoline direct-injection engines, SAE Technical Paper 2019-24-0095 (2019) 1-16 doi:10.4271/2019-24-0095

[50] G. P. Smith, D. M. Golden, M. Frenklach, N. W. Moriarty, B. Eiteneer, M. Goldenberg, C. T. Bowman, R. K. Hanson, S. Song, W. C. Gardiner, Jr., V. V. Lissianski, Z. Qin, Gri-mech 3.0 URL http://www.me.berkeley.edu/gri_mech/

[51] Ö. Gülder, Correlations of laminar combustion data for alternative s.i. engine fuels, SAE Technical Paper 841000 (1984) 1-26 doi : 10.4271/ 841000 